\title{
Soluble selectins and highly fucosylated a1-antichymotrypsin in rheumatoid arthritis patients
}

\author{
Anna Olewicz-Gawlik, Izabela Korczowska-Łącka, Paweł Hrycaj \\ Department of Rheumatology and Clinical Immunology, Poznan University of Medical Sciences, Poland
}

\begin{abstract}
Introduction. Fucosylation of acute phase proteins and serum soluble selectin levels is increased in rheumatoid arthritis (RA) patients and can influence leukocyte extravasation.

Aim. The aim of this study was to evaluate the concentration and fucosylation of a1-antichymotrypsin (ACT) in relation to serum concentrations of soluble forms of selectins in RA patients.

Material and methods. Serum samples of 70 RA patients and 30 healthy controls were examined using sandwich enzyme-linked immunosorbent assay (ELISA).

Results. ACT-FR was significantly increased in RA patients when compared to healthy controls $(p<0.001)$ and significantly correlated with serum concentrations of rheumatoid factor (RF) and antibodies against cyclic citrullinated peptides (ACPA) ( $p=0.006, p=0.04$, respectively). Moreover, we found significant correlations between the serum levels of soluble (s)P- and sE-selectin and ACT-FR ( $p=0.008$ and $p=0.03$, respectively) only in male RA patients.

Conclusions. Fucosylation of ACT differs between male and female RA patients and is related to SP- and sE-selectin levels only in men.
\end{abstract}

Keywords: acute phase proteins, fucosylation, rheumatoid arthritis, selectins.

\section{Introduction}

Rheumatoid arthritis (RA) is a systemic, autoimmune inflammatory disease of unknown origin [1]. The hallmark of RA is a chronic inflammation of joints resulting in joint destruction, functional disability and increased mortality of patients [2]. Systemic inflammation, present in majority of RA patients and reflected in symptoms and biochemical tests, is characterized by activation of vascular endothelium, leukocytes and platelets. These activated cells express selectins [3], which play important roles in coordination of leukocyte migration to extravascular sites of inflammation [4]. E-selectin is expressed only by endothelial cell and circulating E-selectin reflects endothelial activation [5] and mediates angiogenesis via its endothelial ligand sialyl LewisX $\left(s e^{x}\right)$ [6]. P-selectin is an adhesion molecule located in the membrane of alpha granules of platelets and in the
Weibel-Palade bodies of endothelial cells, and its soluble (s) form is a clinical marker of platelet activation [5]. L-selectin is expressed by granulocytes, lymphocytes and monocytes and sL-selectin is a regulator of leukocyte attachment to endothelium [7]. Soluble forms of selectins can be detected in circulation, and their raised levels have been reported in RA [8]. All selectins recognize sialylated, fucosylated glycoconjugates expressed on glycoproteins [9]. Recent outcomes emphasized a critical role of post-translational modifications such as glycosylation in the pathophysiology of many autoimmune diseases. Increased fucosylation of many serum proteins, including antibodies against cyclic citrullinated peptides (ACPA), have been observed in RA patients $[10,11]$. The majority of the fucose residues were detected as $\mathrm{sLe}^{\mathrm{x}}$ on a1-acid glycoprotein (AGP), a1-antichymotrypsin (ACT) and haptoglobin [12]. 
ACT is a serine protease inhibitor which rises in inflammatory conditions and has been implicated in the pathology of a number of devastating human diseases including chronic obstructive pulmonary disease, Parkinson's disease and Alzheimer's disease [13]. In RA the serum concentration of ACT was described as a reliable indicator of the mass of inflamed tissue [14]. ACT acts as an inhibitor of leukocyte cathepsin $G$, which is involved in the pathophysiology of RA [15] and has the unique ability amongst serpins to bind to DNA [16].

As sLe $^{\mathrm{x}}$ epitope on fucosylated glycoproteins can act as a ligand for both membrane bound selectins and their circulating forms, fucosylated proteins can interact with soluble selectins and influence their function. In this study we assessed concentration and fucosylation of ACT in relation to serum concentrations of soluble forms of selectins and disease activity in RA patients.

\section{Material and methods}

\section{Patients}

We recruited to the pilot study 70 consecutive patients (52 women, 18 men, age range 25-75 years, mean $54.7 \pm 12.6$ years), all of whom fulfilled the 2010 American College of Rheumatology (ACR) / European League Against Rheumatism (EULAR) criteria for RA [17]. Patients were also grouped as having early RA ( $n=16$ ) or established RA ( $n=54)$, based upon the duration of the disease, defining early RA as patients having a disease duration of less than 2 years and established RA as patients having a disease duration longer than 2 years. Informed consent was obtained from all participants and the study was approved by the Institutional Review Board at Poznan University of Medical Sciences. A protocol of the conducted research conforms to the principles of the World Medical Association's Declaration of Helsinki.

\section{Clinical evaluation and laboratory measurements}

The workup included the demographics of patients, patients' history, a thorough clinical examination together with patients' status/disease activity assessment using Disease Activity Score (DAS 28) [18, 19]. Functional status was evaluated with Polish version of the Health Assessment Questionnaire (HAQ) disability index [20]. The patients were also asked to assess their overall activity of arthritis and pain using visual analog scales (VAS) [21]. In addition, the 66/68-joint count was performed (66 peripheral joints were evaluated for swelling and 68 peripheral joints were evaluated for tenderness or pain on motion).
Sera from patients were collected at the time of clinical examination and stored at $-70^{\circ} \mathrm{C}$ before estimations were made. The control sera from 30 healthy blood donors (20 women, 10 men, age groups: 25-34 years, 35-44 years, 45-54 years, 55-65 years) were purchased from Regional Blood Center to be analyzed individually. The samples were also pooled and stored at $-70^{\circ} \mathrm{C}$ to be used as a normal control in the lectin enzyme-linked immunosorbent assay (ELISA). Erythrocyte sedimentation rate (ESR) was determined by routine method. Serum C-reactive protein (CRP), AGP and $\mathrm{ACT}$ concentrations were measured by rocket immunoelectrophoresis [22]. The serum SL-, sP-, sE-selectin levels and the concentrations of IgM rheumatoid factor (RF) and ACPA were measured using commercially available ELISA kits (R\&D Systems, USA for selectins and Euroimmun, Germany for IgM RF and ACPA), according to the manufacturer's instructions. The fucosylation of ACT were determined by the Aleuria aurantia lectin (AAL) ELISA, as described previously [23]. The fucosylation ratio (ACT-FR) was calculated as the ratio of the mean sample absorbance to the mean absorbance of the normal pool, after blank subtraction. All samples were assessed in duplicate.

\section{Statistical analysis}

Patients' demographic data were analyzed using descriptive statistics. All continuous data were tested for normal distribution using the Kolmogorov-Smirnov test. The results of analysis of normally distributed data were presented as mean \pm standard deviation (SD), whereas non-normally distributed data were expressed as median (interquartile range, IQR). Differences between investigated groups were tested for significance using a Mann-Whitney U-test. Correlations between variables within the group were analyzed using Spearman's rank-order correlation coefficient (r). Results were considered significant at $p<0.05$ two-sided. All statistical analyses were performed with STATISTICA software (Statsoft, 2009. Statistica version 9.1, www.statsoft.com).

\section{Results}

Table 1 shows the characteristics of RA patients.

Sixty four (91.4\%) patients received disease-modifying anti-rheumatic drugs (DMARDs): 48 (68.6\%) patients received methotrexate, $12(17.1 \%)$ were treated with sulphasalazine, 3 (4.3\%) with leflunomide and $1(1.4 \%)$ with chloroquine. Forty two (60\%) patients 
were treated with glucocorticoids (daily mean dose equivalent to prednisone was $5.6 \mathrm{mg}$ ), from whom 30 received both glucocorticoids and methotrexate. Two women were taking oral contraceptives at the time of examination.

Fucosylation of ACT was significantly lower in healthy controls when compared to RA patients $(0.9(0.5)$ vs. $1.5(1.9), p<0.001$, Figure 1$)$. It was not associated with age or disease duration of the investigated subjects. In RA patients ACT-FR was significantly higher in men than in women $(p=0.01$, Figure 2). ACT-FR significantly correlated with serum concentrations of RF and ACPA ( $r=0.32, p=0.006$ and $r=0.25, p=0.04$, respectively). The correlation between ACT-FR and ACPA was more significant in the group of RA patients not treated with glucocorticoids

Table 1. Demographic, clinical and laboratory characteristics of rheumatoid arthritis patients

\begin{tabular}{lc}
\hline Sex (women/men) & $52 / 18$ \\
\hline Age (years), mean (range) & $54.7 \pm 12.6(25-75)$ \\
\hline Duration of the disease (years), mean (range) & $10.5 \pm 11.4(0,5-56)$ \\
\hline IgM rheumatoid factor present, $n$ (\%) & $60(85.7)$ \\
\hline anti-cyclic citrullinated peptide antibodies present, $n$ (\%) & $48(68.6)$ \\
\hline Health Assessment Questionnaire disability index & $1.4(0.9)$ \\
\hline Visual analog scale pain, mm & $46.5(32)$ \\
\hline DAS 28 (4 variables) & $5.3(1.9)$ \\
\hline DAS 28 (3 variables) & $5.2(1.9)$ \\
\hline Number of tender joints & $21(24.5)$ \\
\hline Number of swollen joints & $12(11.5)$ \\
\hline Erythrocyte sedimentation rate, mm/h & $20(35)$ \\
\hline C-reactive protein, mg/l & $3(9.2)$ \\
\hline SE-selectin (ng/ml) & $24.7(16.9)$ \\
\hline sP-selectin (ng/ml) & $47.3(63)$ \\
\hline sL-selectin (ng/ml) & $1909.1(1022.5)$ \\
\hline a1-antichymotrypsin fucosylation ratio (ACT-FR) & $1.5(1.9)$ \\
\hline
\end{tabular}

Data presented as the median (interquartile range), mean \pm standard deviation or as $n(\%)$

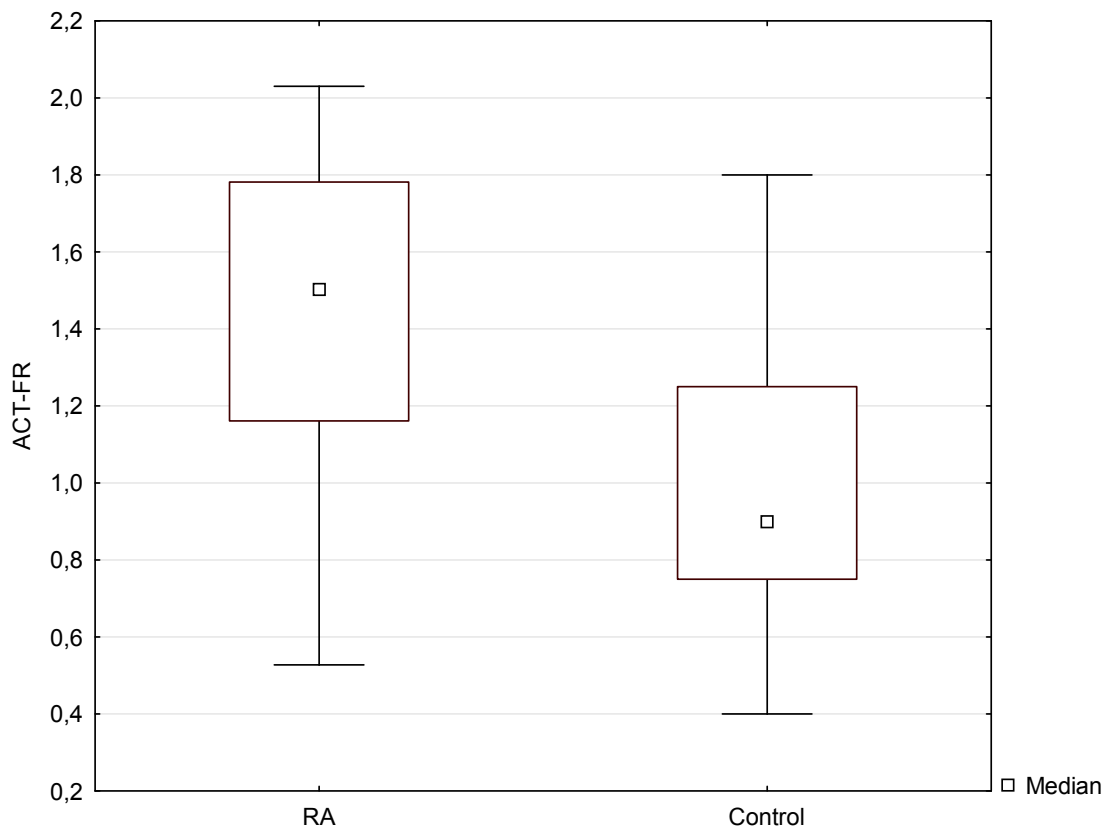

Figure 1. a1-Antichymotrypsin fucosylation ratio (ACT-FR) in rheumatoid arthritis (RA) patients and healthy control (Control) 
$(r=0.46, p=0.01)$. Moreover, ACT-FR and sE-selectin levels were significantly lower in patients with negative RF vs. positive RF ( $p=0.02)$. We noted statistically significant correlation of ACT-FR with sP-selectin ( $r=0.6$, $p=0.008$, Figure 3$)$ and $s E-$ selectin $(r=0.5, p=0.03$, Figure 4) only in males with RA. No correlation was observed between ACT-FR and sL-selectin level.
No other statistically significant associations were detected between ACT-FR and clinical or laboratory findings, as shown in Table 1.We also noted no statistically significant differences in ACT-FR and soluble selectin levels between the group with early vs. established RA or between patients undergoing different immunosuppressive therapies.

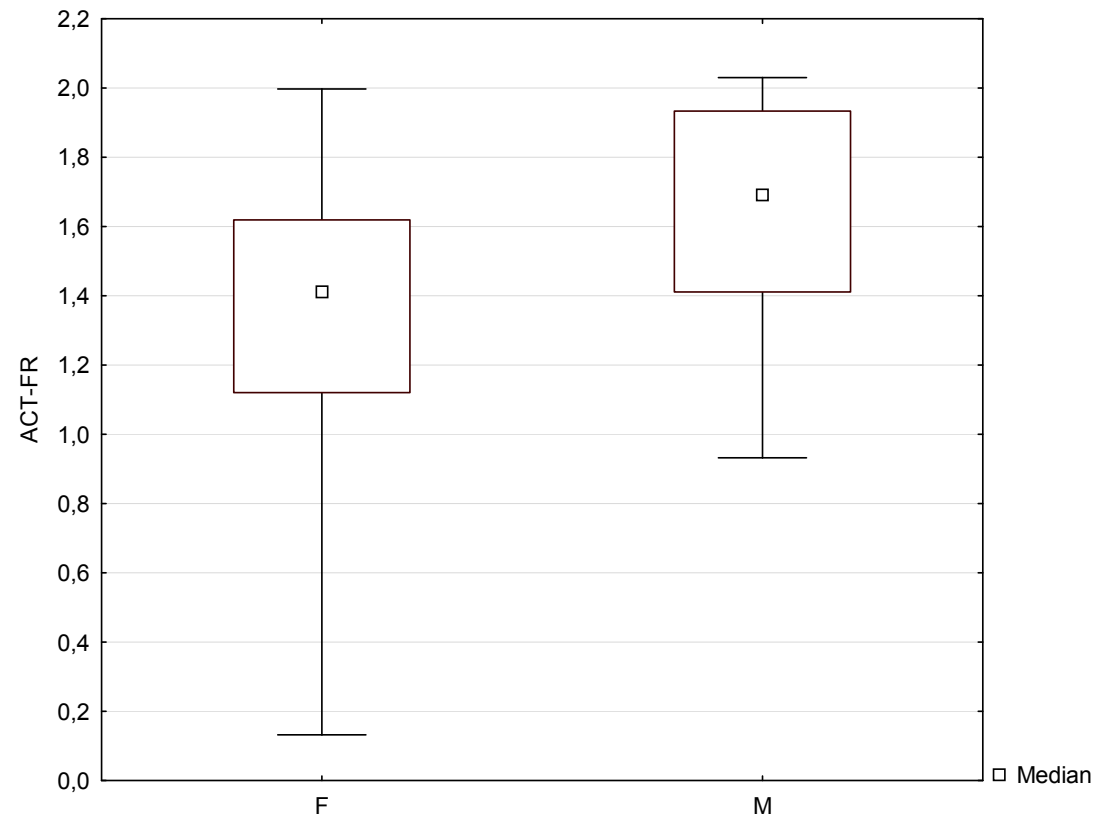

Figure 2. a1-Antichymotrypsin fucosylation ratio (ACT-FR) in female (F) and male (M) rheumatoid arthritis patients

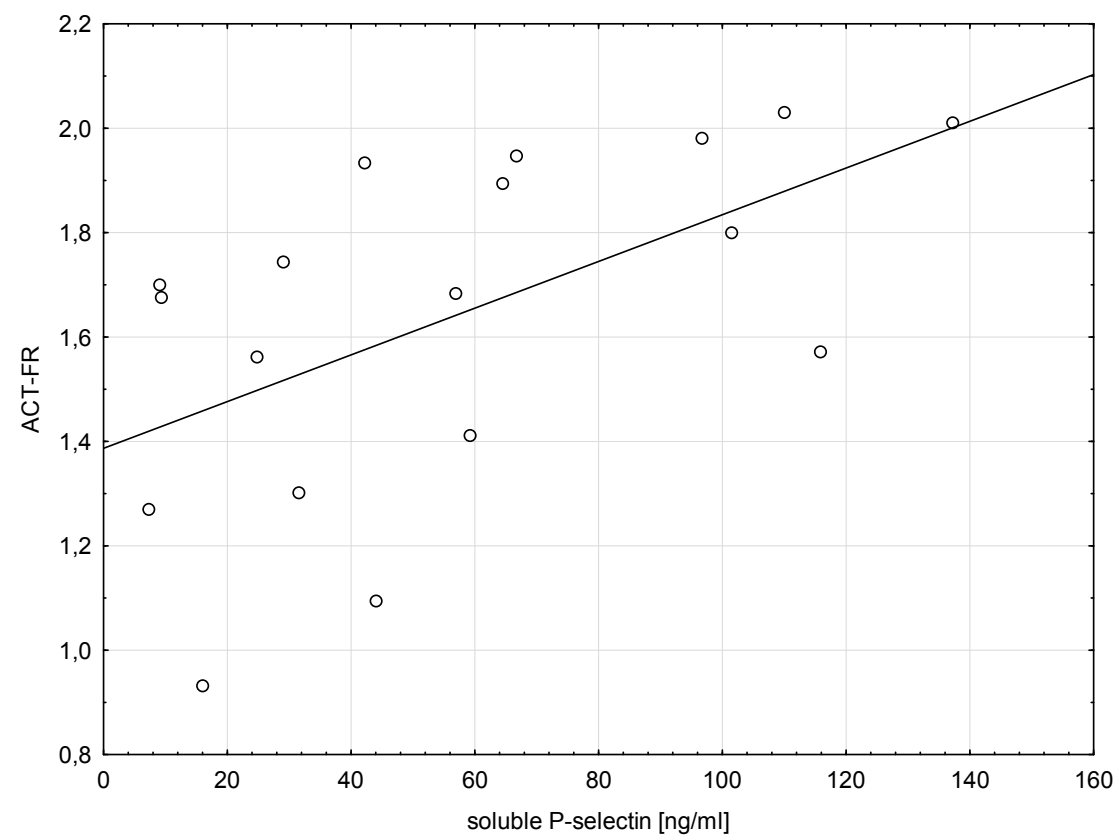

Figure 3. Correlation between a1-antichymotrypsin fucosylation ratio (ACT-FR) and soluble P-selectin in male patients with rheumatoid arthritis 


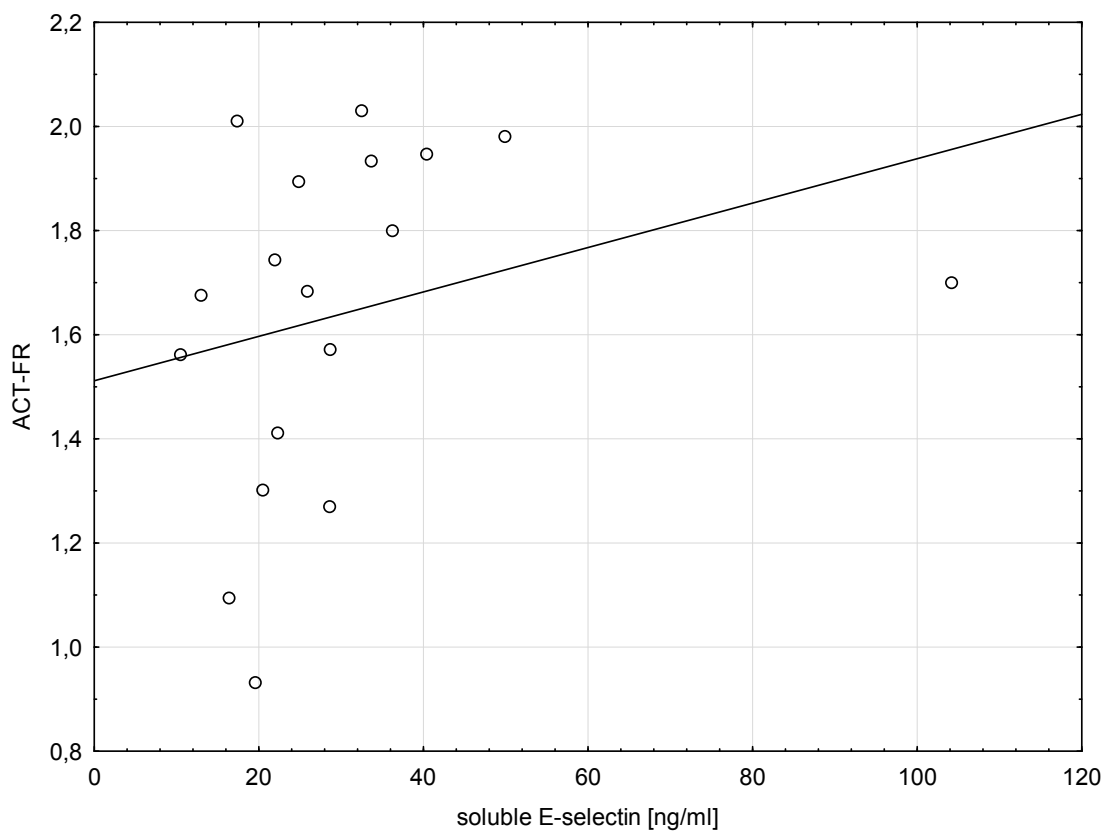

Figure 4. Correlation between a1-antichymotrypsin fucosylation ratio (ACT-FR) and soluble E-selectin in male patients with rheumatoid arthritis

\section{Discussion}

The function of raised levels of circulating selectins and increased fucosylation of glycoproteins in RA patients is obscure. Since sLe ${ }^{x}$ is known to be the simplest carbohydrate structure recognized by the selectins, glycoproteins that present $\mathrm{SLe} \mathrm{e}^{\mathrm{x}}$ may function as the competitive inhibitors of interactions between selectins and their ligands during inflammatory reactions. Thus, the fucosylated proteins may represent a feedback mechanism, leading to the inhibition of leukocyte extravasation by blocking the selectins expressed on activated endothelium. On the other hand, the fucosylated proteins through binding with the soluble forms of selectins could inhibit their possible anti-inflammatory properties. Our hypothesis, that in vivo exists a common mechanism regulating fucosylation of proteins and serum selectin concentrations was promising. As far as we know, this is the first report comparing the fucosylation ratio of ACT with the concentrations of soluble forms of selectins in RA patients.

First of all, in the present study ACT-FR were significantly higher in men than in women, and only in male RA patients statistically significant correlations of ACT-FR with serum concentrations of SP- and sE-selectins were observed. These findings can be a result of altered fucosylation of glycoproteins due to hormonal factors [24]. Moreover, the considerably different results obtained for both sexes could be connect- ed with the distinct RA phenotype between men and women $[25,26]$. Our results are also concordant with previous report concerning fucosylation of a1-acid glycoprotein (AGP-FR) in RA [27]. In the study, Ryden et al. noted a weak correlation between AGP-FR only in men and concluded, that AGP-FR reflects rather basic inflammatory process than short-term variations of clinically assessed disease activity [27]. However, the limitation of our findings can be the fact that men accounted for the minority of the whole examined group. Therefore, the relevance and biological effect of these discoveries remains to be studied in a larger group of patients.

We also showed that ACT-FR significantly correlated with the concentration of RF and ACPA (especially in patients not treated with glucocorticoids), and was significantly lower in patients RF-negative vs. RF-positive. The last observation is concordant with the data reported by Ryden et al., who found a higher degree of AGP fucosylation in RF-positive women than in RF-negative women [27]. Moreover, our finding could be of a particular importance as it was previously reported that higher ACPA concentrations (particularly in RF-positive patients) are associated with increased disease activity in male patients with RA [28]. Thus, it is tempting to speculate that the level of ACT fucosylation not only indicates basal pro-inflammatory phenotype but can have some predictive value in disease outcome in RA. 


\section{Perspectives}

To conclude, our findings demonstrated differences in fucosylation of ACT between male and female RA patients and revealed a relationship between ACT fucosylation and soluble selectins in men. Taken together, our results advocate towards better understanding of fucosylation mechanisms and their modulatory impact on immune system. It can become necessary to face future individualized therapeutic approaches of RA.

\section{Acknowledgements}

\section{Conflict of interest statement}

The authors declare that there is no conflict of interest in the authorship or publication of contribution.

\section{Funding sources}

There are no sources of funding to declare.

\section{References}

1. Lee DM, Weinblatt ME. Rheumatoid arthritis. Lancet 2001 Sep; 358(9285):903-11.

2. Alamanos Y, Drosos AA. Epidemiology of adult rheumatoid arthritis. Autoimmun Rev 2005 Mar; 4(3):130-36.

3. Tedder TF, Steeber DA, Chen A, Engel P. The selectins: vascular adhesion molecules. FASEB J 1995 Jul; 9(10):866-73.

4. Osborn L. Leukocyte adhesion to endothelium in inflammation. Cell 1990 Jul; 62(1):3-6.

5. Eppihimer MJ, Wolitzky B, Anderson DC, Labow MA, Granger DN. Heterogeneity of expression of E- and P-selectins in vivo. Circ Res. 1996 Sep;79(3):560-9.

6. Koch $A E$, Halloran MM, Haskell CJ, Shah MR, Polverini PJ: Angiogenesis mediated by soluble forms of E-selectin and vascular cell adhesion molecule-1. Nature 1995 Aug; 376(6540):517-19.

7. Schleiffenbaum B, Spertini O, Tedder TF. Soluble L-selectin is present in human plasma at high levels and retains functional activity. J Cell Biol. 1992 Oct;119(1):229-38.

8. Ates A, Kinikli G, Turgay M, Duman M. Serum-soluble selectin levels in patients with rheumatoid arthritis and systemic sclerosis. Scand J Immunol 2004 Mar; 59(3):315-20.

9. Foxall C, Watson SR, Dowbenko D et al. The three members of the selectin receptor family recognize a common carbohydrate epitope, the sialyl Lewis(x) oligosaccharide. J Cell Biol 1992 May; 117(4):895-902.

10. Rombouts $Y$, Ewing E, van de Stadt LA et al. Anti-citrullinated protein antibodies acquire a pro-inflammatory Fc glycosylation phenotype prior to the onset of rheumatoid arthritis. Ann Rheum Dis. 2015 Jan;74(1):234-41.

11. Roy S, Biswas S, Saroha A, Sahu D, Das HR. Enhanced expression and fucosylation of ficolin3 in plasma of RA patients. Clin Biochem. 2013 Jan;46(1-2):160-3.

12. Brinkman-van der Linden EC, de Haan PF, Havenaar EC, van Dijk W. Inflammation-induced expression of sialyl LewisX is not restricted to a1-acid glycoprotein but also occurs to a lesser extent on a1-antichymotrypsin and haptoglobin. Glycoconj J 1998 Feb;15(2):177-82.

13. Baker C, Belbin O, Kalsheker N, Morgan K. SERPINA3 (alpha-1-antichymotrypsin). Front Biosci. 2007 May;12:2821-35.

14. Chard MD, Calvin J, Price CP, Cawston TE, Hazleman BL. Serum alpha 1 antichymotrypsin concentration as a marker of disease activity in rheumatoid arthritis. Ann Rheum Dis. 1988 Aug;47(8):665-71.

15. Miyata J, Tani K, Sato K et al. Cathepsin G: the significance in rheumatoid arthritis as a monocyte chemoattractant. Rheumatol Int. 2007 Feb;27(4):375-82.

16. Naidoo N, Cooperman BS, Wang ZM, Liu XZ, Rubin H. Identification of lysines within alpha 1-antichymotrypsin important for DNA binding. An unusual combination of DNA-binding elements. J Biol Chem 1995 Jun; 270(24):14548-55.

17. Aletaha D, Neogi T, Silman AJ et al. 2010 Rheumatoid arthritis classification criteria: an American College of Rheumatology/European League Against Rheumatism collaborative initiative. Arthritis Rheum 2010 Sep; 62(9):2569-81.

18. Van der Heijde DM, van 't Hof M, van Riel PL, van de Putte LB. Development of a disease activity score based on judgment in clinical practice by rheumatologists. J Rheumatol 1993 Mar; 20(3):579-81.

19. Prevoo ML, van 't Hof MA, Kuper HH, van Leeuwen MA, van de Putte LB, van Riel PL. Modified disease activity scores that include twenty-eight-joint counts. Development and validation in a prospective longitudinal study of patients with rheumatoid arthritis. Arthritis Rheum 1995 Jan; 38(1):44-48.

20. Fries JF, Spitz P, Kraines RG, Holman HR. Measurement of patient outcome in arthritis. Arthritis Rheum 1980 Feb; 23(2):137-45.

21. Bird HA, Dixon JS. The measurement of pain. Baillieres Clin Rheumatol 1987 Apr; 1(1):71-89.

22. Laurell CB. Quantitative estimation of proteins by electrophoresis in agarose gel containing antibodies. Analytical Biochemistry 1966 Apr; 15(1):45-52.

23. Ryden I, Lundblad A, Pahlsson P. Lectin ELISA for analysis of $a(1)$-acid glycoprotein fucosylation in the acute phase response. Clin Chem 1999 Nov; 45(11):2010-12.

24. Brinkman-Van der Linden CM, Havenaar EC, Van Ommen CR, Van Kamp GJ, Gooren LJ, Van Dijk W. Oral estrogen treatment induces a decrease in expression of sialyl Lewis $x$ on a 1-acid glycoprotein in females and male-to-female transsexuals. Glycobiology 1996 Jun; 6(4):407-412.

25. Forslind K, Hafström I, Ahlmén M, Svensson B, the BARFOT Study Group. Sex: a major predictor of remission in early rheumatoid arthritis? Ann Rheum Dis 2007 Jan; 66(1):46-52.

26. Tengstrand B, Ahlmén M, Hafström I. The influence of sex on rheumatoid arthritis: a prospective study of onset and outcome after 2 years. J Rheumatol 2004 Feb; 31(2):214-222.

27. Ryden I, Pahlsson P, Lundblad A, Skogh T. Fucosylation of a1-acid glycoprotein (orosomucoid) compared with traditional biochemical markers of inflammation in recent onset rheumatoid arthritis. Clin Chim Acta 2002 Mar; $317(1-2): 221-29$. 
28. Miriovsky BJ, Michaud K, Thiele GM, et al. Anti-CCP antibody and rheumatoid factor concentrations predict greater disease activity in men with rheumatoid arthritis. Ann Rheum Dis. 2010 Jul;69(7):1292-7.

Acceptance for editing: 2015-02-19

Acceptance for publication: 2015-03-30 Repartment of Rheumatology and Clinical Immunology, Poznan University of Medical Sciences, Przybyszewskiego 39, 60-356 Poznan, Poland tel. +48 (61) 8547210, fax +48 (61) 8547212, e-mail anolegaw@ump.edu.p 